\title{
Long non-coding RNA SNHG20 promotes bladder cancer via activating the Wnt/ $\beta$-catenin signalling pathway
}

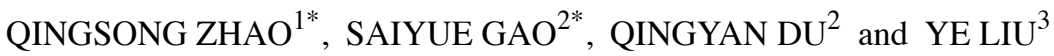 \\ ${ }^{1}$ Department of Urology, Jining No. 1 People's Hospital, Jining, Shandong 272011; \\ ${ }^{2}$ Physical Examination Laboratory, Lanshan District Center for Disease Control and Prevention, Linyi, \\ Shandong 276000; ${ }^{3}$ Clinical Laboratory, Jining No. 1 People's Hospital, Jining, Shandong 272011, P.R. China
}

Received April 5, 2018; Accepted August 6, 2018

DOI: $10.3892 / \mathrm{ijmm} .2018 .3819$

\begin{abstract}
The long non-coding RNA, small nucleolar RNA host gene 20 (SNHG20), is involved in promoting several common types of human cancer, however, the exact function of SNHG20 in the pathogenesis of bladder cancer remains to be elucidated. The present study aimed to examine the regulatory mechanism of SNHG20 underlying the malignant progression of bladder cancer. Reverse transcription-quantitative polymerase chain reaction and western blotting were used to examine mRNA and protein expression. Cell survival, proliferation, apoptosis, colony formation, migration and invasion were also studied. The resulting data indicated that SNHG20 was significantly upregulated in bladder cancer tissues and cell lines, compared with its expression in adjacent non-tumour tissues and the SV-HUC-1 normal urinary tract epithelial cell line, respectively. In addition, the high expression of SNHG20 was associated with advanced clinical stage, lymph node metastasis, and reduced patient survival rate. The knockdown of SNHG20 caused a significant reduction in cancer cell survival, proliferation, colony formation, migration and invasion, and induced cell apoptosis. Additionally, the inhibition of SNHG20 reduced tumour growth in vivo. Investigations into the mechanism revealed that the inhibition of SNHG20 suppressed the activation of Wnt/ $/$-catenin signalling and the expression of certain key genes in bladder cancer cells. Taken together, these results indicated that SNHG20 is involved in promoting bladder cancer and may be used as a potential therapeutic target for the treatment of this disease.
\end{abstract}

Correspondence to: Dr Ye Liu, Clinical Laboratory, Jining No. 1 People's Hospital, 6 Jiankang Road, Jining, Shandong 272011, P.R. China E-mail: liuye272011@163.com

${ }^{*}$ Contributed equally

Key words: bladder cancer, SNHG20, Wnt/ $\beta$-catenin signalling pathway

\section{Introduction}

Bladder cancer is one of the most common types of cancer in humans worldwide and causes a large number of cases of cancer-associated mortality each year $(1,2)$. Rapid tumour growth and metastasis are the main reasons for the high mortality rates of patients with bladder cancer $(2,3)$. Therefore, investigating the regulatory mechanisms underlying the proliferation, migration and invasion of bladder cancer cells may assist in developing novel therapeutic strategies for this disease $(2,3)$.

As a class of non-coding RNAs greater than 200 nucleotides in length, long non-coding RNAs (lncRNAs) function mainly through their interaction with mRNAs, microRNAs (miRs) or proteins (4). LncRNAs have been implicated in a variety of cellular biological processes, including cell proliferation, apoptosis, differentiation, motility, and tumourigenesis (5-7). In addition, certain lncRNAs regulate the expression of genes involved in tumour-related signalling pathways, including Wnt/ $\beta$-catenin and mammalian target of rapamycin/phosphoinositide 3-kinase $(8,9)$. In previous years, an increasing number of studies have reported that IncRNAs, including MALAT1 (10), XIST (11), ATB (12) and GAS5, are key in the development and malignant progression of various types of human cancer, including bladder cancer (13).

The small nucleolar RNA host gene 20 (SNHG20) lncRNA is located on 17q25.2 and contains 2,183 nucleotides. Several studies have reported that SNHG20 is involved in promoting several common types of human cancer, including hepatocellular carcinoma (HCC) $(14,15)$, non-small cell lung cancer (NSCLC) (16), colorectal cancer (17), ovarian cancer (18), gastric cancer (19) and breast cancer (20). For example, SNHG20 was found to be significantly upregulated in HCC and colorectal cancer, and the high expression of SNHG20 was a predictor of poor prognosis $(15,17)$. Chen et al reported that SNHG20 promoted NSCLC cell proliferation and migration by epigenetically silencing the expression of P21 (16). Liu et al found that SNHG20 promoted gastric cancer progression by inhibiting the expression of $\mathrm{p} 21$ and regulating the glycogen synthase kinase-3 $\beta / \beta$-catenin signalling pathway (19). However, the expression and function of SNHG20 in bladder cancer remains to be elucidated.

In the present study, the clinical significance of the expression of SNHG20 in bladder cancer was investigated, and the 
function and molecular mechanism of SNHG20 in regulating the malignant phenotypes of bladder cancer cells were examined.

\section{Materials and methods}

Tissue sample collection. The present study was approved by the Ethics Committee of The First People's Hospital of Jining City (Jining, China). Primary bladder cancer tissues and paired adjacent tumour tissues were collected from 54 patients with bladder cancer at The First People's Hospital of Jining City between March 2011 and September 2012. These patients included 33 men and 21 women, who ranged in age between 43 and 69 years old with a mean age of 60.5 years old. The patients were not exposed to chemotherapy or radiotherapy prior to surgery, and these tissues were confirmed by histopathological evaluation. Informed consent was obtained from all patients. The fresh tissues were stored at $80^{\circ} \mathrm{C}$ until use.

Plasmid construction. To generate the SNHG20 short hairpin (sh)RNA plasmid, self-complementary hairpin DNA oligonucleotides (forward, 5'-GATCCGGCCCAGATT GGTACATTT-3' and reverse, 5'-AGCTTAAATGTACCA ATCTGGGCC-3') were annealed and subcloned into the pRNAT-U6.1/Neo vector (GenScript, Nanjing, China). A negative control was also subcloned into the pRNAT-U6.1/Neo vector (NC shRNA).

Cell culture and transfection. The HT-1376, RT112, 253J, and T24 bladder cancer cell lines and the SV-HUC-1 normal urinary tract epithelial cell line were purchased from the Chinese Academy of Sciences Cell Bank (Shanghai, China). The cells were cultured in Dulbecco's modified Eagle's medium (DMEM, Thermo Fisher Scientific, Inc., Waltham, USA) with $10 \%$ foetal bovine serum (FBS, Thermo Fisher Scientific, Inc.) and incubated at $37^{\circ} \mathrm{C}$ in a $5 \% \mathrm{CO}_{2}$ humidified incubator. For cell transfection, the $253 \mathrm{~J}$ and $\mathrm{T} 24$ cells were cultured to $70 \%$ confluence and transfected with NC shRNA or SNHG20 shRNA using Lipofectamine 2000 (Thermo Fisher Scientific, Inc.) following the manufacturer's protocol. The T24 cells were stably transfected with NC shRNA or SNHG20 shRNA using $400 \mu \mathrm{g} / \mathrm{ml}$ neomycin selection.

Reverse transcription-quantitative polymerase chain reaction (RT-qPCR) analysis. Total RNA was extracted from the cells and tissues using a TRIzol kit (Thermo Fisher Scientific, Inc.), and cDNA was synthesised using a reverse transcription kit (Thermo Fisher Scientific, Inc.). qPCR was performed using a fluorescence quantitative PCR kit (Thermo Fisher Scientific, Inc.) using $1 \mu \mathrm{g}$ cDNA. The reaction conditions for all $\mathrm{qPCR}$ experiments were as follows: $95^{\circ} \mathrm{C}$ for $5 \mathrm{~min}$, followed by 40 cycles of $95^{\circ} \mathrm{C}$ for $10 \mathrm{sec}, 60^{\circ} \mathrm{C}$ for $30 \mathrm{sec}$ and $72^{\circ} \mathrm{C}$ for $30 \mathrm{sec}$. GAPDH was used as the internal reference. The relative expression was analysed using the $2^{-\Delta \Delta \mathrm{Cq}}$ method (21). The sequences of the SNHG20 primers were as follows: Sense, 5'-ATGGCTATAAATAGATACACGC-3' and antisense, 5'-GGTACAAACAGGGAGGGA-3'; the sequences of the GAPDH primers were as follows: Sense, 5'-TGTTCGTCA TGGGTGTGAAC-3' and antisense, 5'-ATGGCATGGACT GTGGTCAT-3'.
Cell survival assay. The transfected cells $(5,000$ cells/well) were seeded in a 96-well plate and incubated for $0,24,48$ or $72 \mathrm{~h}$. Subsequently, $10 \mu \mathrm{l}$ MTT solution $(5 \mathrm{mg} / \mathrm{ml})$ was added. The cells were incubated at $37^{\circ} \mathrm{C}$ for $4 \mathrm{~h}$. Following this, the supernatant was removed, and $100 \mu \mathrm{l}$ of dimethyl sulfoxide was added. The optical density (OD) value at $570 \mathrm{nM}$ was measured on a microplate reader (Bio-Rad Laboratories, Inc., Hercules, CA, USA).

Cell proliferation assay. Cell proliferation was examined using Cell Counting Kit-8 (CCK-8; Dojindo Molecular Technologies, Inc., Kumamoto, Japan). At 48 h post-transfection, the cells (3,000 cells per well) were seeded into 96 -well plates and cultured for $0,24,48$ and $72 \mathrm{~h}$. The OD value at $450 \mathrm{nM}$ was measured on a microplate reader (Bio-Rad Laboratories, Inc.).

Colony formation assay. The transfected cells (500 cells/well) were added to 6-well plates to culture for 14 days and stained with $0.5 \%$ crystal violet (Beyotime Institute of Biotechnology, Haimen, China) at room temperature for $10 \mathrm{~min}$. The cells were then counted and images were captured under an inverted microscope.

Cell apoptosis assay. Following transfection for $48 \mathrm{~h}$, the cells were collected by centrifugation at $1,500 \mathrm{x}$ g for $10 \mathrm{~min}$ at room temperature, and incubated with $500 \mu \mathrm{l}$ binding buffer, $5 \mu \mathrm{l}$ FITC Annexin V and $5 \mu \mathrm{l}$ propidium iodide. The fluorescence of the stained cells was then analysed using flow cytometry (BD FACSCalibur, BD Biosciences, Franklin Lakes, NJ, USA).

Wound healing assay. The bladder cancer cells were grown in 6-well plates with DMEM with 10\% FBS. Wounds were created by scratching the cell surface with a $10-\mu 1$ pipette tip. Then, cells were washed by PBS and cultured at $37^{\circ} \mathrm{C}$ with $5 \% \mathrm{CO}_{2}$ for $48 \mathrm{~h}$. Cells cultured with DMEM served as the blank control group. After $24 \mathrm{~h}$, the cells were observed under an inverted microscope.

Cell invasion assay. Matrigel pre-coated Transwell chambers (BD Biosciences) were used to examine cell invasion. The cell suspension $\left(1 \times 10^{5}\right.$ cells per $\left.\mathrm{ml}\right)$ was prepared in DMEM, following which, $300 \mu \mathrm{l}$ of DMEM with $10 \%$ FBS was added into the lower chamber, and $300 \mu \mathrm{l}$ of cell suspension was added into the upper chamber. The cells were then cultured at $37^{\circ} \mathrm{C}$ for $24 \mathrm{~h}$, and cells that did not invade through the membrane in the filter were removed by wiping. The cells that had invaded through the membrane were fixed, stained with crystal violet (Sigma-Aldrich; Merck KGaA, Darmstadt, Germany), and counted under an inverted microscope.

Tumour formation assay. BALB/c mice $(\mathrm{n}=8$, male, 20-22 g, 8-week-old, Hunan SJA Laboratory Animal Co., Ltd, Changsha, China) were maintained under specific pathogen-free conditions: Free access to food and water at $22-25^{\circ} \mathrm{C}$ under a $12 \mathrm{~h}$ light/dark cycle. T2 4 cells were stably transfected with SNHG20 shRNA or NC shRNA, and a cell suspension containing $10^{7}$ cells was subcutaneously injected into the posterior flank of each animal. The tumour volumes were determined at different time points (tumour 


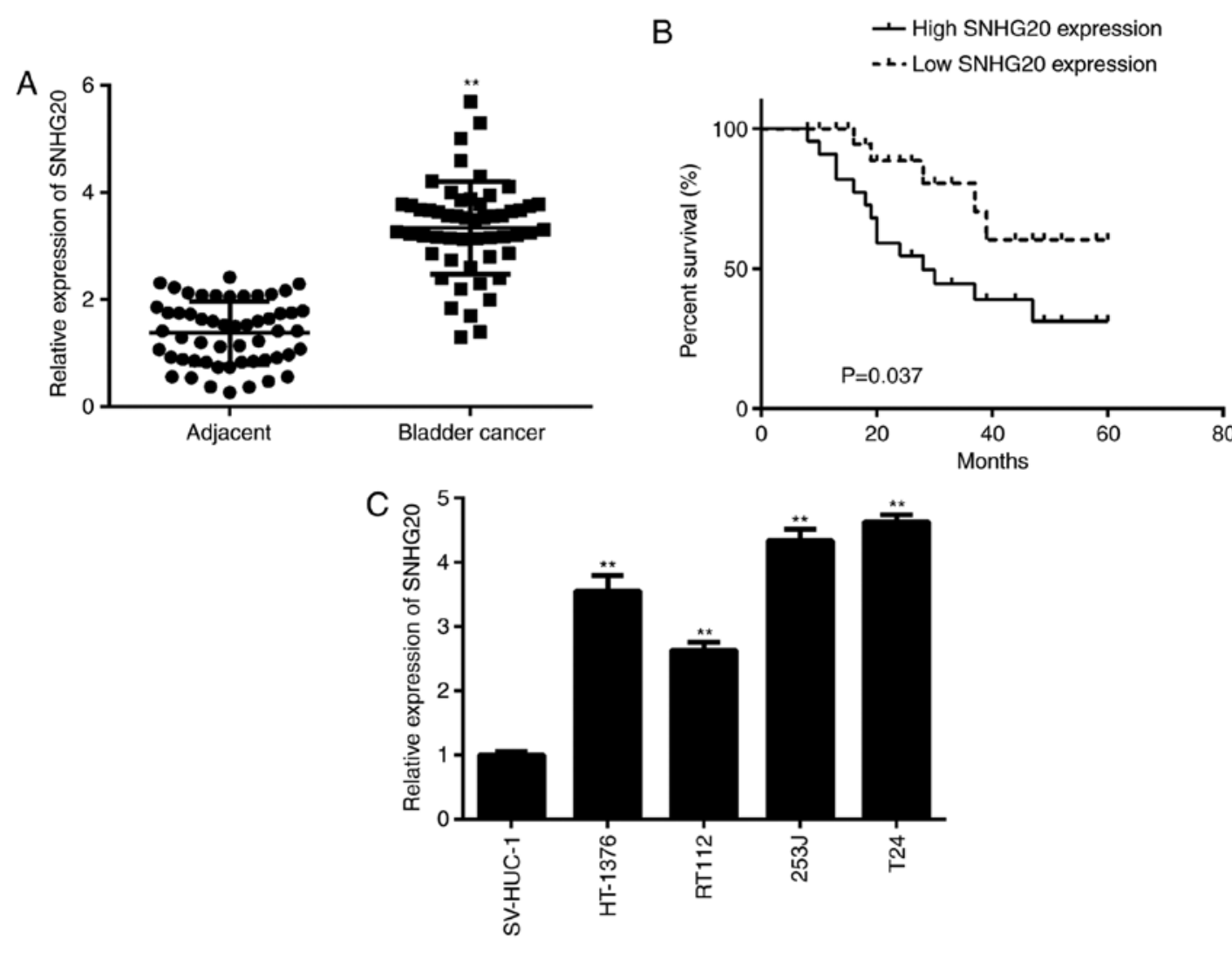

Figure 1. SNHG20 is upregulated in bladder cancer tissues. (A) RT-qPCR analysis was used to examine the expression of SNHG20 in bladder cancer tissues and matched adjacent non-tumour tissues. ${ }^{* * *} \mathrm{P}<0.01$, vs. Adjacent. (B) Patients with bladder cancer with a high expression of SNHG20 had reduced survival rates, compared with those with a low expression of SNHG20. (C) RT-qPCR analysis was used to examine the expression of SNHG20 in bladder cancer cell lines and the SV-HUC-1 normal urinary tract epithelial cell line. ${ }^{* *} \mathrm{P}<0.01$, vs. SV-HUC-1. SNHG20, small nucleolar RNA host gene 20; RT-qPCR, reverse transcription-quantitative polymerase chain reaction.

volume = length $\mathrm{x}$ width $\left.{ }^{2} \mathrm{x} 0.5\right)$. At 30 days following injection, all animals were sacrificed, and tumour tissues were obtained.

Western blot analysis. Total proteins were extracted from cells using RIPA lysis buffer (Thermo Fisher Scientific, Inc.) and protein concentration was determined using the Pierce BCA Protein Assay kit (Thermo Fisher Scientific, Inc.). The proteins (50 $\mu \mathrm{g}$ per lane) were separated on $10 \%$ SDS-PAGE gels and then transferred onto PVDF membranes (Thermo Fisher Scientific, Inc.). The membranes were blocked with $5 \%$ non-fat dry milk at room temperature for $3 \mathrm{~h}$ and then incubated with primary antibodies, including antibodies targeting Caspase-3 (1:200, cat. no. ab13847, Abcam, Cambridge, MA, USA), Caspase-9 (1:200, cat. no. ab32539, Abcam), B-cell lymphoma 2 (Bcl2; 1:500, cat. no. ab32124, Abcam), matrix metalloproteinase (MMP)2 (1:200, cat. no. ab92536, Abcam), MMP9 (1:500, cat. no. ab76003, Abcam), c-Myc (1:200, cat. no. ab32072, Abcam), $\beta$-catenin (1:200, cat. no. ab16051, Abcam), and GAPDH (1:200, cat. no. ab9485, Abcam) at room temperature for $3 \mathrm{~h}$. Subsequently, the membranes were incubated with HRP-conjugated secondary antibody (1:5,000, cat. no. ab6721, Abcam) for $1 \mathrm{~h}$ at room temperature. Chemiluminescence was examined using SuperSignal West Femto Maximum Sensitivity substrate (Thermo Fisher Scientific, Inc.). The quantities of protein were analysed using ImageJ software 1.46 (National Institutes of Health, Bethesda, MD, USA). GAPDH was used as the internal control.
Statistical analysis. Data are expressed as the mean \pm standard deviation. SPSS 20.0 software (IBM SPSS, Armonk, NY, USA) was used for statistical analysis. Student's t-test was used for comparisons between two groups, and one-way analysis of variance followed by Tukey's post hoc test was used for comparisons of more than two groups. The Kaplan-Meier method was used for survival analysis. $\mathrm{P}<0.05$ was considered to indicate a statistically significant difference.

\section{Results}

SNHG20 is upregulated in bladder cancer tissues. In the present study, the expression of SNHG20 was first examined in bladder cancer tissues and matched adjacent non-tumour tissues. The RT-qPCR data revealed that SNHG20 was significantly upregulated in the bladder cancer tissues compared with the matched adjacent non-tumour tissues (Fig. 1A). The association between the expression of SNHG20 and clinicopathological characteristics in patients with bladder cancer was then examined. Based on the mean expression value of SNHG20 in bladder cancer tissues, the patients were divided into a low expression group and high expression group. As shown in Table I, a high expression of SNHG20 was significantly associated with advanced tumour-node-metastasis (TNM) stage and lymph node metastasis in bladder cancer. In addition, it was observed that patients with bladder cancer with a high expression of SNHG20 exhibited reduced survival 

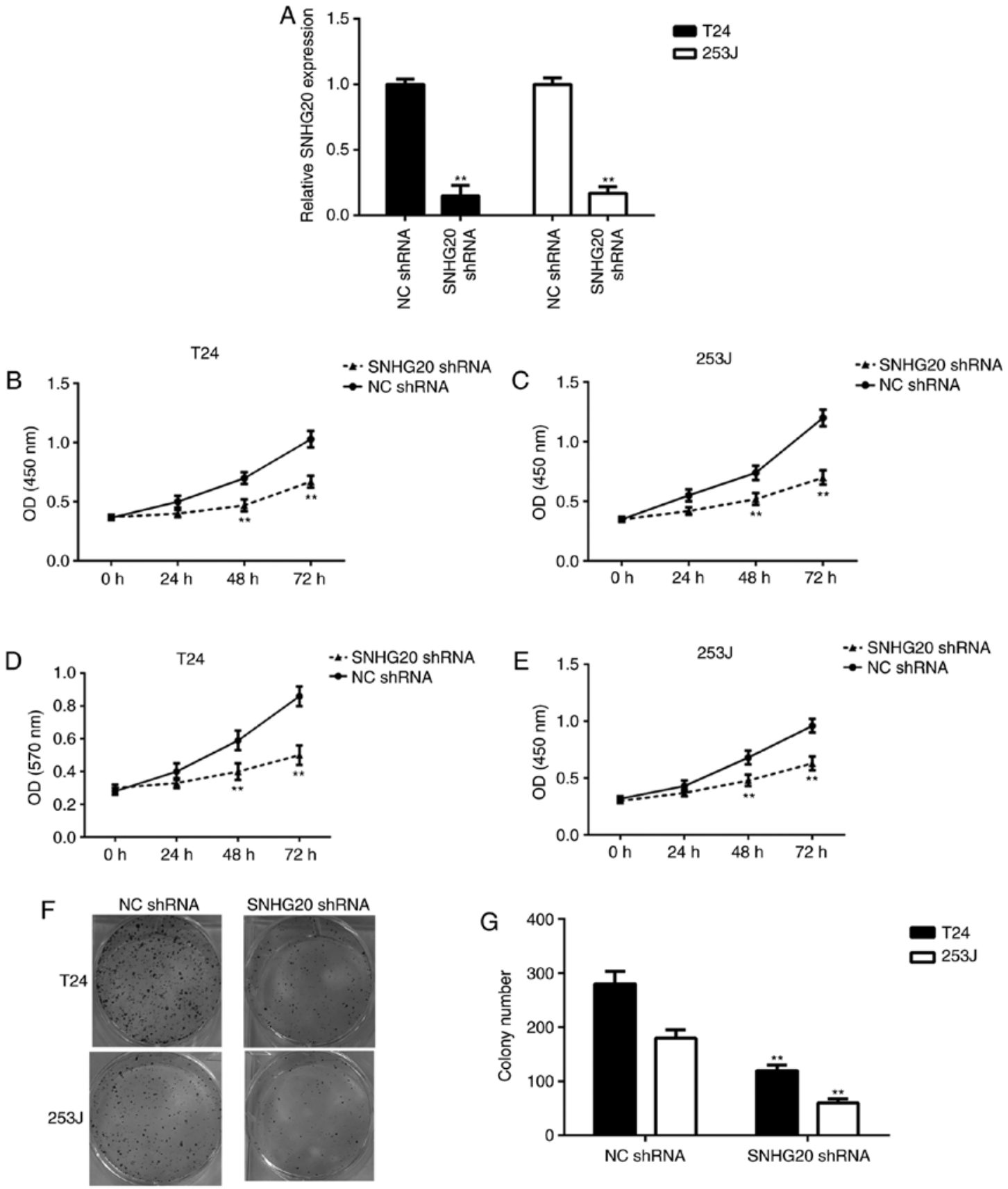

Figure 2. Knockdown of SNHG20 inhibits bladder cancer cell proliferation, survival and colony formation. T24 and 253J cells were transfected with NC shRNA or SNHG20 shRNA. Following transfection, (A) reverse transcription-quantitative polymerase chain reaction analysis was used to examine the expression of SNHG20. A Cell Counting Kit-8 assay was performed to examine (B) T24 and (C) 253J cell proliferation. An MTT assay was performed to examine (D) T24 and (E) 253J cell survival. (F) Colony formation capacities of the cells were examined and (G) quantified. ${ }^{* *} \mathrm{P}<0.01$, vs. NC shRNA. SNHG20, small nucleolar RNA host gene 20; shRNA, short hairpin RNA; NC, negative control; OD, optical density.

rate compared with those with a low expression of SNHG20 (Fig. 1B). In addition, the expression of SNHG20 was detected in bladder cancer cell lines (HT-1376, RT112, 253J, and T24) and the SV-HUC-1 normal urinary tract epithelial cell line. The data indicated that the expression of SNHG20 was increased in the bladder cancer cell lines compared with its expression in the SV-HUC-1 cells (Fig. 1C). Therefore, it was suggested that SNHG20 is upregulated in bladder cancer, contributing to its malignant progression and poor prognosis.

SNHG20 knockdown inhibits bladder cancer cell proliferation and survival, and induces cell apoptosis. To investigate the function of SNHG20 in bladder cancer, the T24 and 253J bladder cancer cells were transfected with SNHG20 shRNA or NC shRNA, separately. Following transfection, the RT-qPCR data showed that the expression of SNHG20 was significantly reduced in the SNHG20 shRNA group compared with that in the NC shRNA group (Fig. 2A). The CCK-8 assay and MTT assay data showed that the knockdown of SNHG20 significantly reduced the proliferation and survival of bladder cancer cells (Fig. 2B-E). In addition, the downregulation of SNHG20 reduced the colony formation ability of the bladder cancer cells (Fig. 2F and G). Therefore, inhibiting the expression of SNHG20 reduced bladder cancer cell proliferation and survival. 

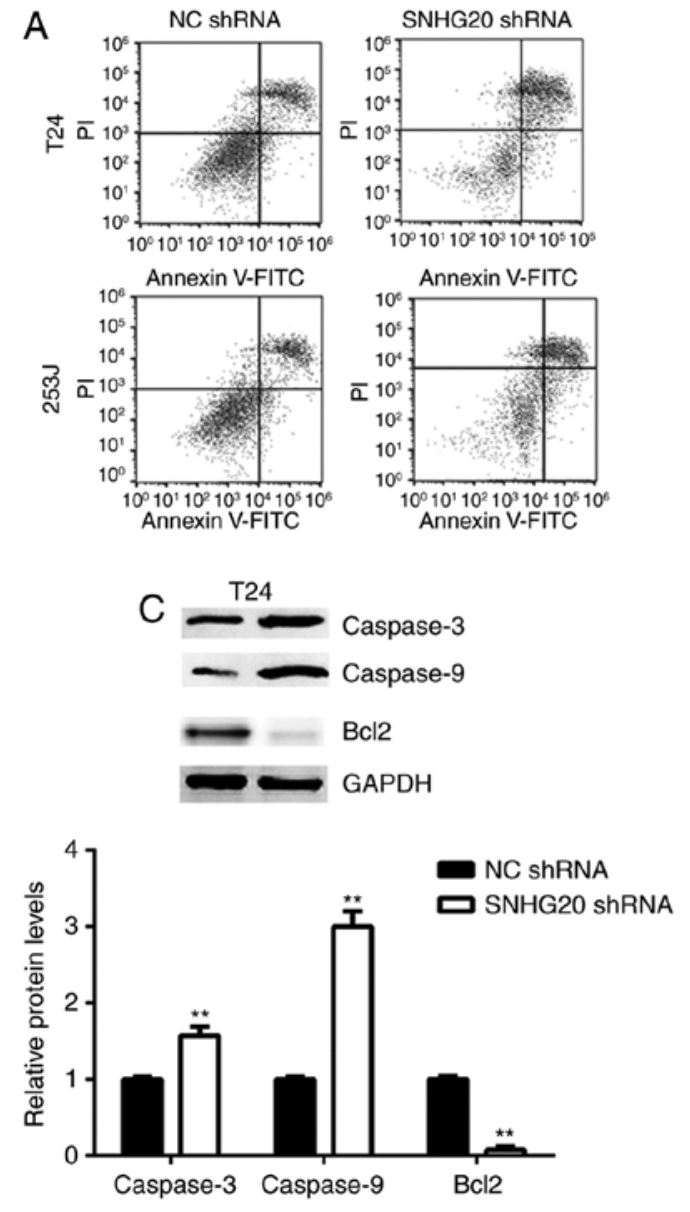
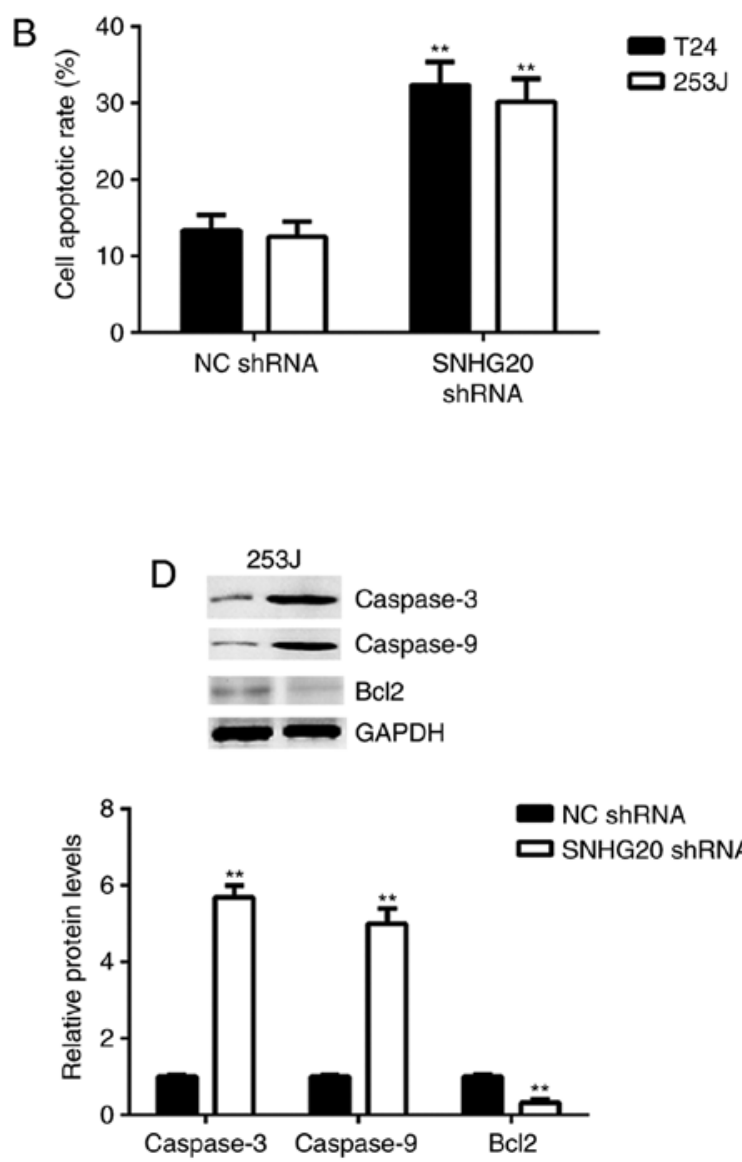

Figure 3. Knockdown of SNHG20 induces bladder cancer cell apoptosis. T24 and 253J cells were transfected with NC shRNA or SNHG20 shRNA. (A) Following transfection, flow cytometry was used to examine cell apoptosis with (B) quantification of results. Western blot analysis was performed to examine the protein expression of Caspase-3, Caspase-9 and Bcl2 in (C) T24 and (D) 253J cells. ${ }^{* *}$ P $<0.01$, vs. NC shRNA. SNHG20, small nucleolar RNA host gene 20; shRNA, short hairpin RNA; NC, negative control; PI, propidium iodide; Bcl2, B-cell lymphoma 2.

Table I. Association between the expression of SNHG20 and clinicopathological characteristics in bladder cancer.

\begin{tabular}{|c|c|c|c|c|}
\hline \multirow[b]{2}{*}{ Characteristic } & \multirow[b]{2}{*}{$\begin{array}{l}\text { Cases } \\
(n=54)\end{array}$} & \multicolumn{2}{|c|}{ Expression of SNHG20 } & \multirow[b]{2}{*}{ P-value } \\
\hline & & $\begin{array}{l}\text { High } \\
(n=28)\end{array}$ & $\begin{array}{c}\text { Low } \\
(n=26)\end{array}$ & \\
\hline Age (years) & & & & 0.574 \\
\hline$<55$ & 20 & 9 & 11 & \\
\hline$\geq 55$ & 34 & 19 & 15 & \\
\hline Sex & & & & 0.586 \\
\hline Male & 33 & 16 & 17 & \\
\hline Female & 21 & 12 & 9 & \\
\hline $\begin{array}{l}\text { Lymph node } \\
\text { metastasis }\end{array}$ & & & & $0.024^{\mathrm{a}}$ \\
\hline Negative & 35 & 14 & 21 & \\
\hline Positive & 19 & 14 & 5 & \\
\hline Stage & & & & $0.014^{\mathrm{a}}$ \\
\hline I-II & 27 & 9 & 18 & \\
\hline III-IV & 27 & 19 & 8 & \\
\hline
\end{tabular}

${ }^{a} \mathrm{P}<0.05$ was considered to indicate a statistically significant difference. SNHG20, small nucleolar RNA host gene 20.
The present study then investigated the effects of SNHG20 on bladder cancer cell apoptosis. Flow cytometric assay data indicated that SNHG20 knockdown significantly induced bladder cancer cell apoptosis compared with the cells transfected with the NC shRNA (Fig. 3A and B). Consistently, SNHG20 knockdown increased the protein expression levels of Caspase- 3 and Caspase- 9 and inhibited the protein expression of Bcl2 in the bladder cancer cells (Fig. 3C and D).

Inhibition of the expression of SNHG2O decreases the migration and invasion of bladder cancer cells. Tumour cell migration and invasion are key processes during cancer metastasis. Therefore, the present study examined whether SNHG20 affected the migration and invasion of bladder cancer cells. The wound healing assay data revealed that bladder cancer cell migration was significantly repressed in the SNHG20 shRNA group compared with that in the NC shRNA group (Fig. 4A and B). The Transwell assay data indicated that bladder cancer cell invasion was also significantly decreased in the SNHG20 shRNA group compared with that in the NC shRNA group (Fig. 4C and D). Consistently, the protein levels of MMP2 and MMP9, two key factors in tumour metastasis, were significantly downregulated following SNHG20 knockdown (Fig. 4E and F). These findings demonstrated that inhibition of the expression of SNHG20 decreased the migration and inva- 
A
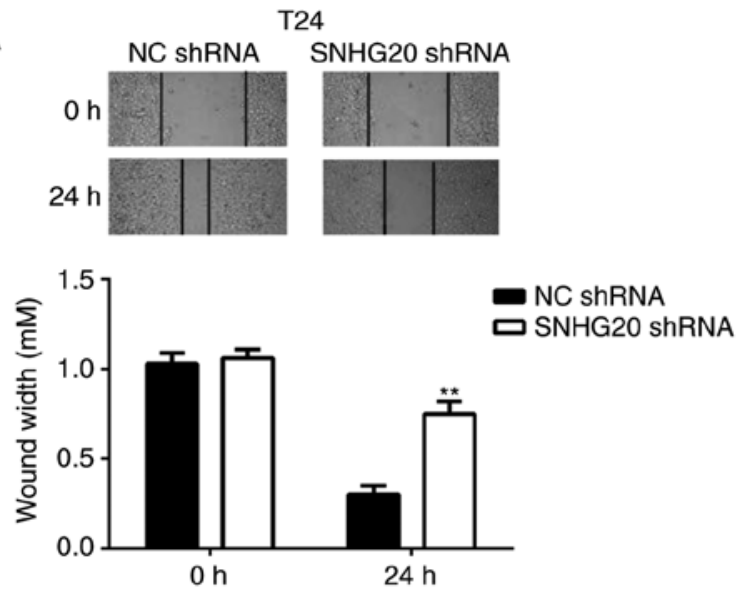

C
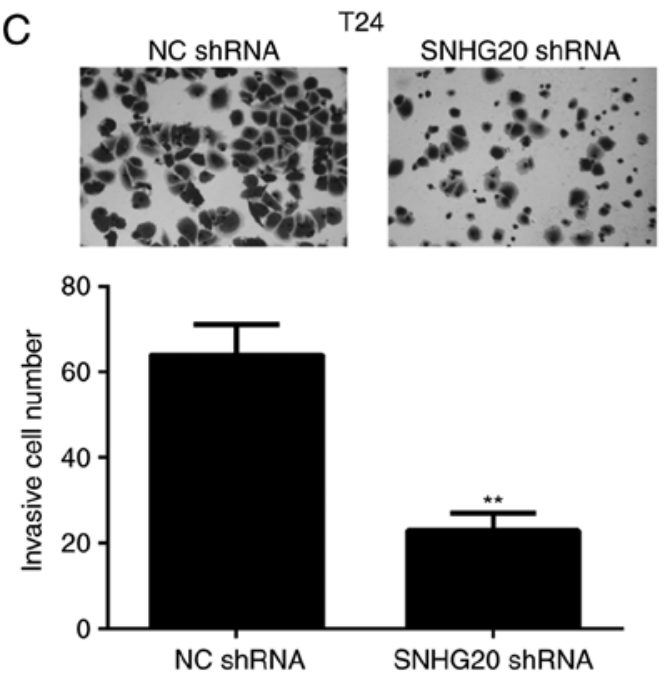

E
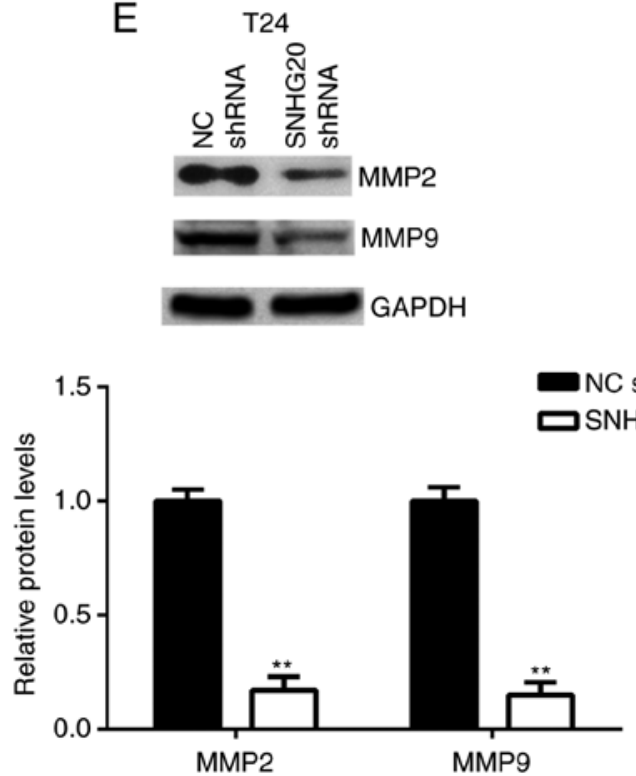

B
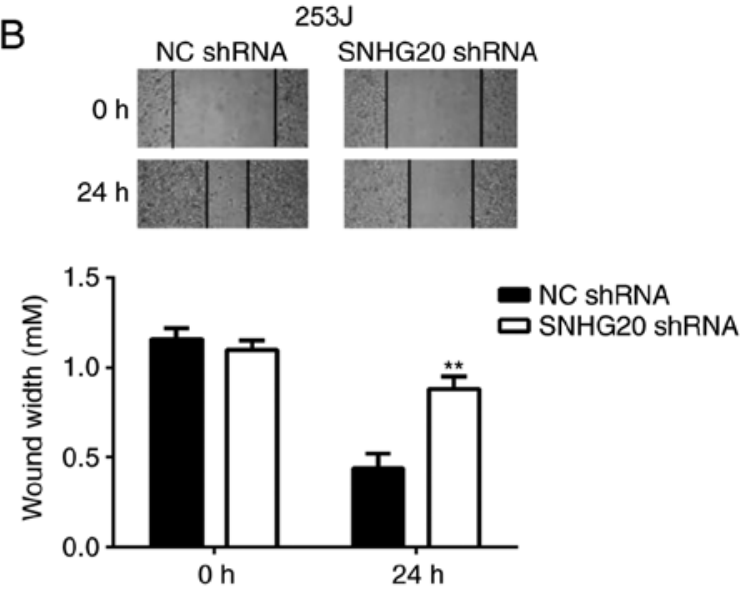

D
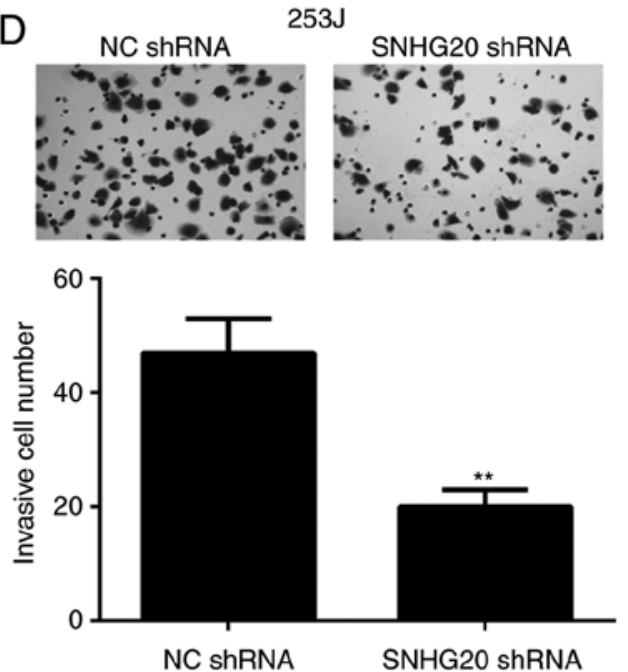

$\mathrm{F}$
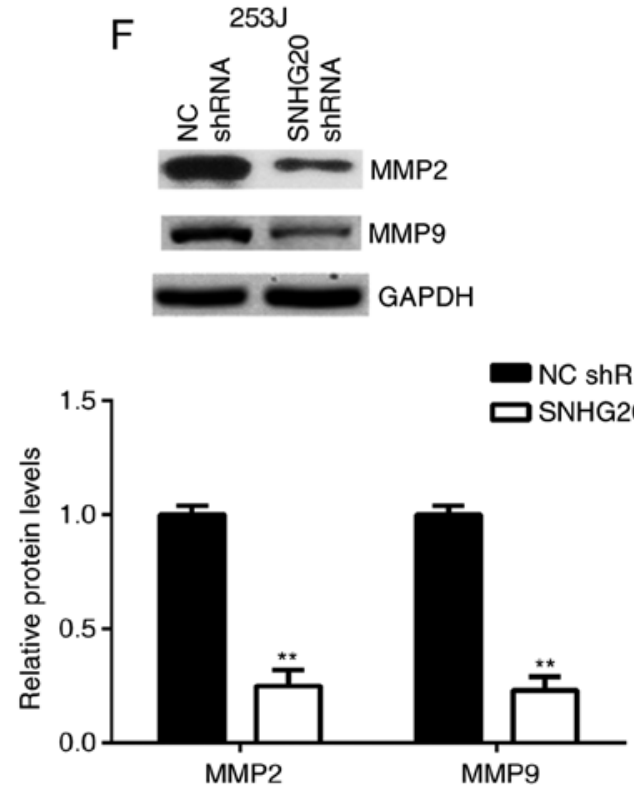

Figure 4. Inhibition of the expression of SNHG20 decreases the migration and invasion of bladder cancer cells. T24 and 253J cells were transfected with NC shRNA or SNHG20 shRNA. Following transfection, wound healing assay was used to examine (A) T24 and (B) 253J cell migration. A Transwell assay was used to examine (C) T24 and (D) 253J cell invasion (magnification, x400). Western blot analysis was performed to examine the protein expression of MMP2 and MMP9 in (E) T24 and (F) 253J cells. ${ }^{* *}$ P<0.01, vs. NC shRNA. SNHG20, small nucleolar RNA host gene 20; shRNA, short hairpin RNA; NC, negative control; MMP, matrix metalloproteinase.

sion of bladder cancer cells and suggested that SNHG20 may be involved in promoting cancer metastasis.
Knockdown of SNHG2O inhibits Wnt/ $\beta$-catenin signalling pathway activity. Wnt/ $\beta$-catenin signalling is key in the 
A
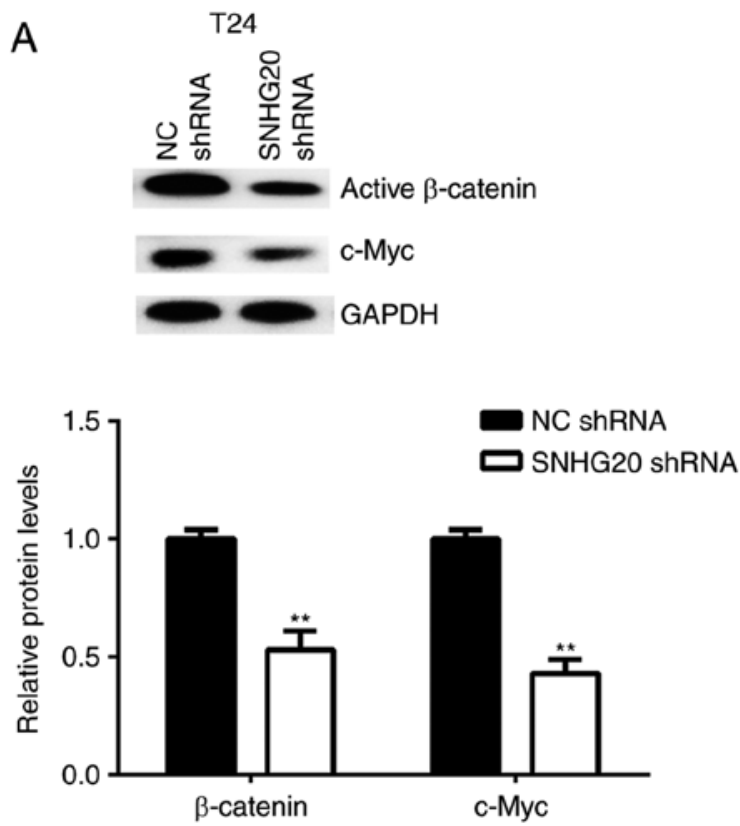

B

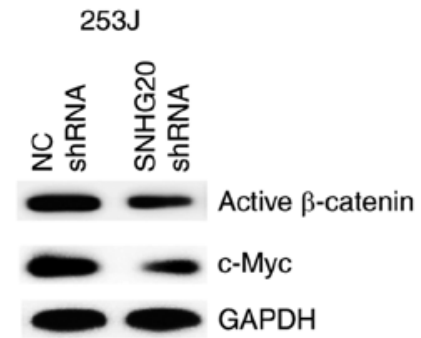

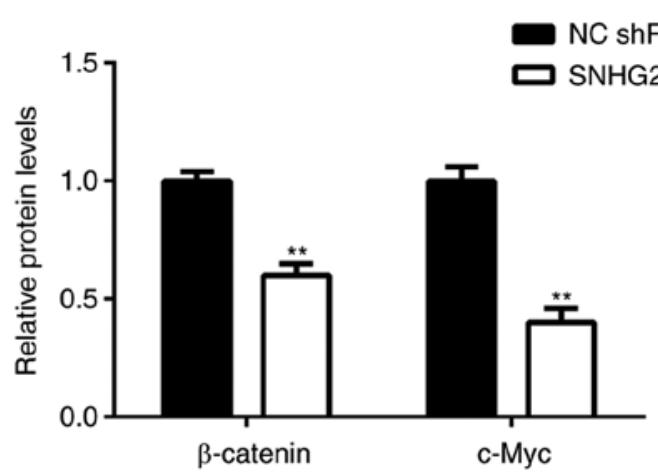

Figure 5. Knockdown of SNHG20 inhibits the activity of the Wnt/ $\beta$-catenin signalling pathway. T24 and 253J cells were transfected with NC shRNA or SNHG20 shRNA. Following transfection, western blot analysis was performed to examine the protein expression of active $\beta$-catenin and c-Myc in (A) T24 and (B) 253J cells. ** P<0.01, vs. NC shRNA. SNHG20, small nucleolar RNA host gene 20; shRNA, short hairpin RNA; NC, negative control.

pathogenesis of bladder cancer. Therefore, the present study examined the effects of the downregulation of SNHG20 on $\mathrm{Wnt} / \beta$-catenin signalling activity in bladder cancer cells. The protein levels of active $\beta$-catenin and c-Myc, a key target gene of $\mathrm{Wnt} / \beta$-catenin signalling, were examined in bladder cancer cells following SNHG20 knockdown. The western blot data showed that the protein levels of active $\beta$-catenin and c-Myc were significantly reduced in the SNHG20 shRNA group compared with the levels in the NC shRNA group (Fig. 5A and B). Therefore, the knockdown of SNHG20 inhibited $\mathrm{Wnt} / \beta$-catenin signalling pathway activity.

SNHG20 knockdown inhibits tumour growth of bladder cancer cells in vivo. Finally, the effects of SNHG20 in bladder cancer were examined in vivo. A BALB/c nude mouse xenograft model was established using T24 cells that were stably transfected with SNHG20 shRNA. The data revealed that the tumour volumes and weights were significantly reduced in the SNHG20 shRNA group compared with those in the NC shRNA group (Fig. 6A-C). Subsequently, xenograft tissues were obtained and the expression of SNHG20 was examined in each group. The RT-qPCR data confirmed that the levels of SNHG20 were reduced in the SNHG20 shRNA group compared with those in the NC shRNA group (Fig. 6D). In addition, the protein levels of MMP2, MMP9, active $\beta$-catenin and c-Myc were reduced in the SNHG20 shRNA group compared with those in the NC shRNA group (Fig. 6E and F). These data indicated that SNHG20 knockdown inhibited the tumour growth of bladder cancer cells in a mouse xenograft model and was associated with reduced Wnt/ $\beta$-catenin signalling activity.

\section{Discussion}

Investigating the regulatory mechanisms underlying bladder cancer growth and metastasis may be beneficial for the devel- opment of promising therapeutic strategies for bladder cancer. In the present study, it was found that SNHG20 was significantly upregulated in bladder cancer tissues and cell lines, compared with its expression in adjacent non-tumour tissues and the SV-HUC-1 normal urinary tract epithelial cell line, respectively. In addition, the high expression of SNHG20 was associated with advanced clinical stage, lymph node metastasis, and reduced survival rate of patients. SNHG20 knockdown caused a significant reduction in cancer cell survival, proliferation, colony formation, migration and invasion, and induced cell apoptosis. The inhibition of SNHG20 also reduced tumour growth in vivo. In addition, the inhibition of SNHG20 suppressed the activation of $\mathrm{Wnt} / \beta$-catenin signalling and the expression of certain key genes in bladder cancer cells.

In previous years, several lncRNAs have been demonstrated to be dysregulated in bladder cancer and involved in its malignant progression $(22,23)$. For example, lncRNA ATB promotes the proliferation, migration and invasion of bladder cancer cells by suppressing miR-126 (12). LncRNA SPRY4-IT1 sponges miR-101-3p to promote the proliferation and metastasis of bladder cancer cells through increasing the expression of enhancer of zeste homolog 2 (EZH2) (24). However, the expression and exact role of SNHG20 in bladder cancer remains to be fully elucidated. In the present study, it was found that the expression levels of SNHG20 were significantly higher in bladder cancer tissues than in matched adjacent non-tumour tissues, and its increased expression was significantly associated with advanced TNM stage, lymph node metastasis, and reduced survival rates of patients with bladder cancer. These findings suggested that the upregulation of SNHG20 may contribute to the malignant progression of bladder cancer and that SNHG20 may serve as a potential predicator for the prognosis of patients with bladder cancer.

As SNHG20 was significantly upregulated in bladder cancer, bladder cancer cells were transfected with SNHG20 

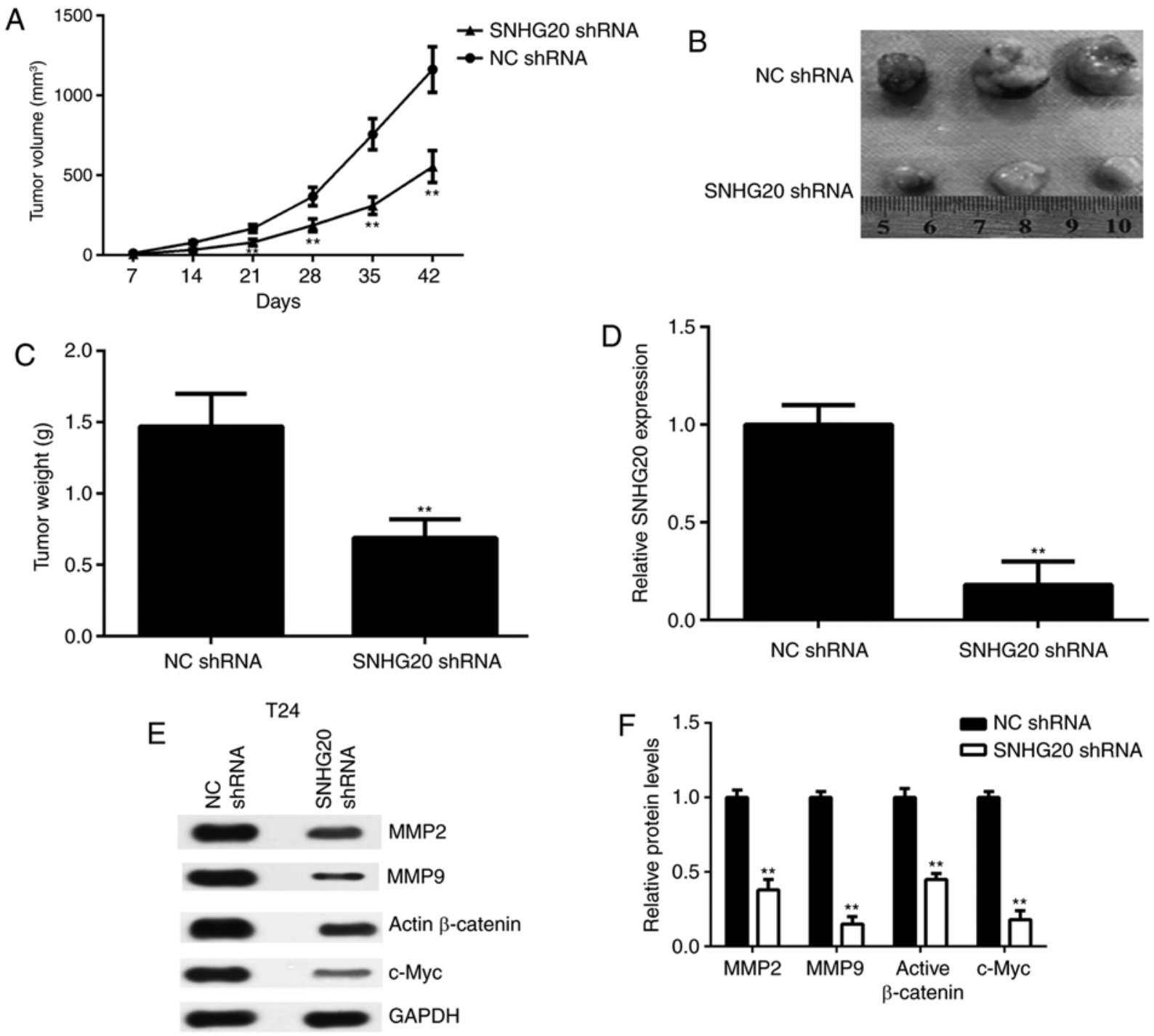

Figure 6. Knockdown of SNHG20 inhibits tumour growth of bladder cancer cells in vivo. T24 cells were stably transfected with SNHG20 shRNA or NC shRNA, and subcutaneously injected into the posterior flank of the nude mice. (A) Tumour volume was examined. (B) Images of the tumour tissues were captured. (C) Tumour weight was determined. (D) Reverse transcription-quantitative polymerase chain reaction analysis was used to examine the expression of SNHG20 in the tumour tissues. (E) Western blot analysis was performed to examine the protein expression of MMP2, MMP9, active $\beta$-catenin and c-Myc. (F) Quantification of protein levels. ${ }^{* *} \mathrm{P}<0.01$, vs. NC shRNA. SNHG20, small nucleolar RNA host gene 20; shRNA, short hairpin RNA; NC, negative control; MMP, matrix metalloproteinase.

shRNA to knock down its expression. Further investigation revealed that SNHG20 knockdown markedly inhibited the proliferation, survival and colony formation of the bladder cancer cells. In addition, SNHG20 knockdown caused a significant reduction in the tumour growth of bladder cancer cells in a xenograft mouse model. These findings suggested that SNHG20 promoted the proliferation of bladder cancer cells in vitro and in vivo. The effects of the inhibition of SNHG20 on bladder cancer cell apoptosis were then examined. The flow cytometry results revealed that the downregulation of SNHG20 notably induced bladder cancer cell apoptosis. Consistent with these findings, SNHG20 knockdown increased the protein levels of two key apoptotic biomarkers, Caspase-3 and Caspase-9, but decreased the expression of $\mathrm{Bcl} 2$, an important anti-apoptotic protein $(25,26)$.

Tumour cell migration and invasion have been shown to promote tumour growth and enhance cancer invasion and metastasis $(27,28)$. Therefore, the present study examined the role of SNHG20 in the regulation of bladder cancer cell migration and invasion. The findings showed that inhibiting the expression SNHG20 significantly reduced cell migration and invasion, accompanied with decreased expression levels of MMP2 and MMP9, two key factors associated with extracellular matrix degradation and tumour invasion and metastasis (29). These findings suggested that SNHG20 may be involved in promoting bladder cancer metastasis.

It has been widely reported that the expression levels of Wnt factors are significantly upregulated in bladder cancer $(30,31)$, and the Wnt/ $\beta$-catenin signalling is important in the malignant progression of bladder cancer $(32,33)$. For example, Shen et al showed that the levels of $\beta$-catenin in human bladder cancer tissues were upregulated with increasing grade of malignancy (30). Schmitz-Drager et al investigated a total of 185 paraffin-embedded bladder cancer tissue specimens immunohistochemically for the overexpression of c-myc (31). Mao et al reported that activation of the 
Wnt/ $\beta$-catenin signalling pathway induced epithelial-mesenchymal transition and promote bladder cancer metastasis (34). In addition, the Wnt signalling has been suggested as a molecular target for bladder cancer $(35,36)$. For example, Guo et al showed that the downregulation of miR-144 increased bladder cancer cell proliferation by targeting EZH2 and regulating Wnt signalling (35). Costa et al reported that the epigenetic deregulation of Wnt pathway inhibitors contributed to aberrant activation of the Wnt signalling pathway in bladder (36). He et al reported that SNHG20 was involved in promoting ovarian cancer progression by activating the $\mathrm{Wnt} / \beta$-catenin signalling pathway (18). Therefore, the present study investigated whether SNHG20 functioned in bladder cancer through regulating the $\mathrm{Wnt} / \beta$-catenin signalling pathway. The data obtained in the present study showed that inhibiting the expression of SNHG20 in bladder cancer cells caused a significant reduction in the expression levels of active $\beta$-catenin and $\mathrm{c}-\mathrm{Myc}$, a target gene of the Wnt/ $\beta$-catenin signalling (37). These findings suggested that SNHG20 also activates the Wnt/ $\beta$-catenin signalling pathway in bladder cancer cells.

In conclusion, to the best of our knowledge, the present study is the first report of SNHG20 being significantly upregulated in bladder cancer and that this was associated with its malignant progression and poor patient prognosis. In addition, SNHG20 was found to activate the $\mathrm{Wnt} / \beta$-catenin pathway and has a promoting role in bladder cancer. These findings suggested that SNHG20 may become a potential therapeutic target for bladder cancer treatment. Further investigations are required to clarify the function of SNHG20 in bladder cancer metastasis in vivo using animal experiments.

\section{Acknowledgements}

Not applicable.

\section{Funding}

No funding was received.

\section{Availability of data and materials}

All data generated or analysed during the present study are included within this manuscript.

\section{Authors' contributions}

QZ and SG collected clinical tissues and performed experiments. QD performed statistical analysis. QZ wrote the manuscript. YL designed the study and revised the manuscript.

\section{Ethics approval and consent to participate}

This study was approved by the Ethics Committee of The First People's Hospital of Jining City, and written informed consent was obtained from all participants.

\section{Patient consent for publication}

Not applicable.

\section{Competing interests}

The authors declare that they have no competing interests.

\section{References}

1. Siegel RL, Miller KD and Jemal A: Cancer statistics, 2015. CA Cancer J Clin 65: 5-29, 2015.

2. Skeldon SC and Larry Goldenberg S: Bladder cancer: A portal into mens health. Urol Onco 33: 40-44, 2015.

3. Sathe A and Nawroth R: Targeting the PI3K/AKT/mTOR pathway in bladder cancer. Methods Mol Biol 1655: 335-350, 2018.

4. Li J,Zi Y, Wang W and Li Y: LncRNA MEG3 inhibits cell proliferation and metastasis in chronic myeloid leukemia via targeting miR-184. Oncol Res 26: 297-305, 2018.

5. Zhang JJ, Wang DD, Du CX and Wang Y: Long noncoding RNA ANRIL promotes cervical cancer development by acting as a sponge of miR-186. Oncol Res: May 22, 2017 (Epub ahead of print). doi: 10.3727/096504017X14953948675449.

6. Yang M,Zhai X, Ge T, Yang C and Lou G: miR-181a-5p promotes proliferation and invasion, and inhibits apoptosis of cervical cancer cells via regulating inositol polyphosphate-5-phosphatase A (INPP5A). Oncol Res 26: 703-712, 2018.

7. Wang Y, Li J, Xu C and Zhang X: MicroRNA-139-5p inhibit cell proliferation and invasion by targeting RHO-associated coiled-coil containing protein kinase 2 in ovarian cancer. Oncol Res: Jun 14, 2017 (Epub ahead of print) doi: 10.3727/096504017 X14974343584989.

8. Liu L, Yu D, Shi H, Li J and Meng L: Reduced lncRNA aim enhances the malignant invasion of triple-negative breast cancer cells mainly by activating Wnt/beta-catenin/mTOR/PI3K signaling. Pharmazie 72: 599-603, 2017.

9. Jia L, Tian Y, Chen Y and Zhang G: The silencing of LncRNA-H19 decreases chemoresistance of human glioma cells to temozolomide by suppressing epithelial-mesenchymal transition via the Wnt/beta-catenin pathway. Onco Targets Ther 11: 313-321, 2018.

10. Xie H, Liao X, Chen Z, Fang Y, He A, Zhong Y Gao Q, Xiao H, Li J, Huang W and Liu Y: LncRNA MALAT1 inhibits apoptosis and promotes invasion by antagonizing miR-125b in bladder cancer cells. J Cancer 8: 3803-3811, 2017.

11. Hu Y, Deng C, Zhang H, Zhang J, Peng B and Hu C: Long non-coding RNA XIST promotes cell growth and metastasis through regulating miR-139-5p mediated Wnt/beta-catenin signaling pathway in bladder cancer. Oncotarget 8: 94554-94568, 2017.

12. Zhai $\mathrm{X}$ and $\mathrm{Xu} \mathrm{W}$ : Long noncoding RNA ATB promotes proliferation, migration and invasion in bladder cancer by suppressing microRNA-126. Oncol Res: Jan 10, 2018 (Epub ahead of print). doi: $10.3727 / 096504018 X 15152072098476$.

13. Wang M, Guo C, Wang L, Luo G, Huang C, Li Y, Liu D, Zeng $F$,Jiang $G$ and Xiao $X$ : Long noncoding RNA GAS5 promotes bladder cancer cells apoptosis through inhibiting EZH2 transcription. Cell Death Dis 9: 238, 2018.

14. Liu J, Lu C, Xiao M, Jiang F, Qu L and Ni R: Long non-coding RNA SNHG20 predicts a poor prognosis for HCC and promotes cell invasion by regulating the epithelial-to-mesenchymal transition. Biomed Pharmacother 89: 857-863, 2017.

15. Zhang D, Cao C, Liu L and Wu D: Up-regulation of LncRNA SNHG20 predicts poor prognosis in hepatocellular carcinoma. J Cancer 7: 608-617, 2016.

16. Chen Z, Chen X, Chen P, Yu S, Nie F, Lu B, Zhang T, Zhou Y, Chen Q, Wei C, et al: Long non-coding RNA SNHG20 promotes non-small cell lung cancer cell proliferation and migration by epigenetically silencing of P21 expression. Cell Death Dis 8: e3092, 2017.

17. Li C, Zhou L, He J, Fang XQ, Zhu SW and Xiong MM: Increased long noncoding RNA SNHG20 predicts poor prognosis in colorectal cancer. BMC Cancer 16: 655, 2016.

18. He S, Zhao Y, Wang X, Deng Y, Wan Z, Yao S and Shen H: Up-regulation of long non-coding RNA SNHG20 promotes ovarian cancer progression via Wnt/ $\beta$-catenin signaling. Biosci Rep 38, 2018.

19. Liu J, Liu L, Wan JX and Song Y: Long noncoding RNA SNHG20 promotes gastric cancer progression by inhibiting $\mathrm{p} 21$ expression and regulating the GSK-3beta/beta-catenin signaling pathway. Oncotarget 8: 80700-80708, 2017. 
20. Guan YX, Zhang ZM, Chen XZ, Zhang Q, Liu SZ and Zhang YL: Lnc RNA SNHG20 participated in proliferation, invasion and migration of breast cancer cells via miR-495. J Cell Biochem: Dec 13, 2017 (Epub ahead of print). doi: 10.1002/jcb.26588.

21. Livak KJ and Schmittgen TD: Analysis of relative gene expression data using real-time quantitative PCR and the 2(-Delta Delta $\mathrm{C}(\mathrm{T}))$ method. Methods 25: 402-408, 2001.

22. Huang T, Liu HW, Chen JQ, Wang SH, Hao LQ, Liu M and Wang B: The long noncoding RNA PVT1 functions as a competing endogenous RNA by sponging miR-186 in gastric cancer. Biomed Pharmacother 88: 302-308, 2017.

23. Xue M, Pang H, Li X, Li H, Pan J and Chen W: Long noncoding RNA UCA1 promotes bladder cancer cell migration and invasion via hsa-miR-145/ZEB1/2/FSCN1 pathway. Cancer Sci 107: 18-17, 2016.

24. Liu D, Li Y, Luo G, Xiao X, Tao D, Wu X, Wang M, Huang C, Wang L, Zeng F and Jiang G: LncRNA SPRY4-IT1 sponges miR-101-3p to promote proliferation and metastasis of bladder cancer cells through up-regulating EZH2. Cancer Lett 388: 281-291, 2017.

25. Maurya SK, Tewari M, Sharma B and Shukla HS: Expression of procaspase 3 and activated caspase 3 and its relevance in hormone-responsive gallbladder carcinoma chemotherapy. Korean J Intern Med 28: 573-578, 2013.

26. Yang TQ, Lu XJ, Wu TF, Ding DD, Zhao ZH, Chen GL, Xie XS Li B, Wei YX, Guo LC, et al: MicroRNA-16 inhibits glioma cell growth and invasion through suppression of BCL2 and the nuclear factor-kappaB1/MMP9 signaling pathway. Cancer Sci 105: 265-271, 2014.

27. Fife CM, McCarroll JA and Kavallaris M: Movers and shakers: Cell cytoskeleton in cancer metastasis. Br J Pharmacol 171: 5507-5523, 2014

28. Mowers EE, Sharifi MN and Macleod KF: Functions of autophagy in the tumor microenvironment and cancer metastasis. FEBS J 285: 1751-1766, 2018.

29. Kapoor C, Vaidya S, Wadhwan V, Hitesh, Kaur G and Pathak A: Seesaw of matrix metalloproteinases (MMPs). J Cancer Res Ther 12: 28-35, 2016
30. Shen $\mathrm{CH}$, Wu JD, Jou YC, Cheng MC, Lin CT, Chen PC, Tseng YS, Shi CS, Chen SY, Chang DC and Lee YR: The correlation between TWIST, E-cadherin, and beta-catenin in human bladder cancer. J BUON 16: 733-737, 2011.

31. Schmitz-Drager BJ, Schulz WA, Jurgens B, Gerharz CD, van Roeyen CR, Bültel H, Ebert T and Ackermann R: c-myc in bladder cancer. Clinical findings and analysis of mechanism. Urol Res 25 (Suppl 1): S45-S49, 1997.

32. Mao XW, Xiao JQ, Li ZY, Zheng YC and Zhang N: Effects of microRNA-135a on the epithelial-mesenchymal transition, migration and invasion of bladder cancer cells by targeting GSK3beta through the Wnt/beta-catenin signaling pathway. Exp Mol Med 50: e429, 2018.

33. Yuan H, Yu S, Cui Y, Men C, Yang D, Gao Z, Zhu Z and Wu J: Knockdown of mediator subunit Med19 suppresses bladder cancer cell proliferation and migration by downregulating Wnt/beta-catenin signalling pathway. J Cell Mol Med 21: 3254-3263, 2017.

34. Mao XW, Xiao JQ, Xu G, Li ZY, Wu HF, Li Y, Zheng YC and Zhang N: CUL4B promotes bladder cancer metastasis and induces epithelial-to-mesenchymal transition by activating the Wnt/beta-catenin signaling pathway. Oncotarget 8: 77241-77253, 2017.

35. Guo Y, Ying L, Tian Y, Yang P, Zhu Y, Wang Z, Qiu F and Lin J: miR-144 downregulation increases bladder cancer cell proliferation by targeting EZH 2 and regulating Wnt signaling. FEBS J 280: 4531-4538, 2013.

36. Costa VL, Henrique R, Ribeiro FR, Carvalho JR, Oliveira J, Lobo F, Teixeira MR and Jerónimo C: Epigenetic regulation of Wnt signaling pathway in urological cancer. Epigenetics 5: 343-351, 2010.

37. Hu Y, Yu K, Wang G, Zhang D, Shi C, Ding Y, Hong D, Zhang D, $\mathrm{He} \mathrm{H}$, Sun L, et al: Lanatoside $\mathrm{C}$ inhibits cell proliferation and induces apoptosis through attenuating Wnt/beta-catenin/c-Myc signaling pathway in human gastric cancer cell. Biochem Pharmacol 150: 280-292, 2018. 